\title{
ARTICLE \\ Rebalancing of the gut flora and microbial metabolism is responsible for the anti-arthritis effect of kaempferol
}

\author{
Li-xiang $\mathrm{Aa}^{1}$, Fei Fei ${ }^{1}$, $\mathrm{Qi} \mathrm{Qi}{ }^{1}$, Run-bin Sun ${ }^{1}$, Sheng-hua Gu${ }^{2}$, Zi-zhen $\mathrm{Di}^{3}$, Ji-ye Aa ${ }^{1}$, Guang-ji Wang ${ }^{1}$ and Chang-xiao Liu ${ }^{4}$
}

Kaempferol is a natural flavonol that possesses various pharmacological activities, including anti-arthritis effects, yet the underlying mechanisms remain controversial. To evaluate the anti-arthritis efficacy and the underlying mechanisms of kaempferol, collageninduced arthritis (CIA) mice were treated with kaempferol intragastrically $\left(200 \mathrm{mg} \cdot \mathrm{kg}^{-1} \cdot \mathrm{d}^{-1}\right)$ and intraperitoneally $\left(20 \mathrm{mg} \cdot \mathrm{kg}^{-1} \cdot \mathrm{d}^{-1}\right)$. Pharmacodynamic and pharmacokinetic studies showed that the oral administration of kaempferol produced distinct anti-arthritis effects in model mice with arthritis in terms of the spleen index, arthritis index, paw thickness, and inflammatory factors; the bioavailability $\left(1.5 \%\right.$, relative to that of the intraperitoneal injection) and circulatory exposure of kaempferol $\left(C_{\max }=0.23 \pm 0.06 \mathrm{ng} / \mathrm{mL}\right)$ and its primary metabolite kaempferol-3-O-glucuronide $\left(C_{\max }=233.29 \pm 89.64 \mathrm{ng} / \mathrm{mL}\right)$ were rather low. In contrast, the intraperitoneal injection of kaempferol caused marginal anti-arthritis effects, although it achieved a much higher in vivo exposure. The much higher kaempferol content in the gut implicated a potential mechanism involved in the gut. Analysis of 16S ribosomal RNA revealed that CIA caused imbalance of 14 types of bacteria at the family level, whereas kaempferol largely rebalanced the intestinal microbiota in CIA mice. A metabolomics study showed that kaempferol treatment significantly reversed the perturbation of metabolites involved in energy production and the tryptophan, fatty acid and secondary bile acid metabolisms in the gut contents of the CIA mice. In conclusion, we demonstrate for the first time that the high level of kaempferol in the gut regulates the intestinal flora and microbiotic metabolism, which are potentially responsible for the anti-arthritis activities of kaempferol.

Keywords: kaempferol; collagen-induced arthritis; anti-arthritis effects; pharmacokinetics; intestinal microbiota; metabolomics

Acta Pharmacologica Sinica (2020) 41:73-81; https://doi.org/10.1038/s41401-019-0279-8

\section{INTRODUCTION}

Rheumatoid arthritis (RA) is a chronic autoimmune disease accompanied by synovitis, cartilage erosion, and bone destruction [1]. Conventional remedies, such as disease-modifying antirheumatic drugs (DMARDs) and non-steroidal anti-inflammatory drugs (NSAIDs), produce therapeutic effects, but serious and widespread adverse reactions to these drugs have significantly restricted their continuous use [2-4]. Herbal medicines provide an alternative choice to treat RA due to their definite efficacy with fewer side effects [5-7].

Kaempferol is a natural flavonol found in many edible plants and herbal medicines. Kaempferol shows a wide range of pharmacological activities, including anti-inflammatory and antioxidant effects [8-10]. RA is reported to be associated with oxidative stress [11] and high levels of inflammatory mediators [12]. Recently, studies have shown that kaempferol alleviates pathological symptoms in arthritis animal models after oral administration [13]. In an in vitro study, the anti-arthritis activity of kaempferol was shown to regulate the activity of inflammatory enzymes [14], the formation of osteoclasts [15], and the proliferation, migration, and invasion of fibroblast-like synoviocytes $[13,16]$ at an effective concentration of $7.5-100 \mu \mathrm{M}$.
However, our preliminary study revealed that the oral administration of kaempferol had a distinct anti-arthritis effect, while the intraperitoneal injection of kaempferol showed a marginal effect even though the intraperitoneal in vivo exposure of kaempferol was much higher than the exposure of the intragastric route. To assess this peculiarity, collagen-induced model mice were employed to evaluate the pharmacokinetics and pharmacodynamics of kaempferol. Further mechanisms were explored by analyzing the gut microbiota and small molecule metabolites based on a high-throughput transcriptomics and metabolomics.

\section{MATERIALS AND METHODS}

Animals

Male DBA/1J mice (6-7 weeks) were obtained from Cavens Lab Animal Ltd. (Changzhou, Jiangsu, China). The mice were maintained in specific pathogen free conditions and fed regular chow and sterile water under a $12 / 12 \mathrm{~h}$ light-dark cycle. The experimental procedures were conducted strictly according to the guidelines of the Committee on the Care and Use of Laboratory Animals and the related ethical regulations of China Pharmaceutical University.

\footnotetext{
${ }^{1}$ Jiangsu Province Key Laboratory of Drug Metabolism and Pharmacokinetics, State Key Laboratory of Natural Medicines, China Pharmaceutical University, Nanjing 210009, China;

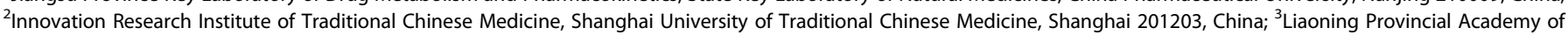

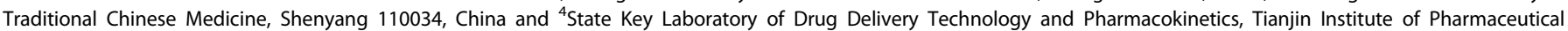
Research, Tianjin 300193, China

Correspondence: Ji-ye Aa (jiyea@cpu.edu.cn) or Chang-xiao Liu (liuchangxiao@163.com)
}

Received: 19 March 2019 Accepted: 23 June 2019

Published online: 19 August 2019 
Collagen-induced arthritis (CIA) model mice

Bovine type II collagen (Cll; Chondrex, Redmond, WA, USA) was emulsified with an equal volume of complete Freund's adjuvant (Sigma-Aldrich, St. Louis, MO, USA). DBA/1J mice were immunized via intradermal injection at the base of the tail with $100 \mu \mathrm{L}$ of emulsion on day 0 as the first immunization. On day 21 after immunization, a booster with $100 \mu \mathrm{L}$ of the same bovine CII emulsified in incomplete Freund's adjuvant (Chondrex, Redmond, WA, USA) was administered by the same route.

For the oral administration study, mice were gavaged daily with kaempferol (purity: 98\%; MUST, Chengdu, China) at a dose of 200 $\mathrm{mg} / \mathrm{kg}$ or leflunomide (purity: $98 \%$ ) at a dose of $5 \mathrm{mg} / \mathrm{kg}$ from day 21 to day 48. For the alternative intraperitoneal injection study, 20 $\mathrm{mg} / \mathrm{kg}$ of kaempferol was injected daily from day 21 to day 48 .

\section{Arthritis assessment}

Each paw score was judged as follows: 0 , no signs of arthritis; 1 , mild swelling confined to the tarsal bones or ankle joint; 2 , mild swelling extending from the ankle to the tarsal bones; 3 , moderate swelling extending from the ankle to the metatarsal joints; and 4, severe swelling encompassing the ankle, foot, and digits or ankylosis of the limb. The scores for each paw were summed to yield a total arthritis severity score per mouse, with a maximum score of 16 for each animal.

\section{Histopathological examination}

On day 48 , the mice were humanely euthanized, and the hind limbs were collected, immersed in $10 \%$ buffered formalin, decalcified in $10 \%$ EDTA, and embedded in paraffin. Sections (5 $\mu \mathrm{m}$ ) of the joints were stained with hematoxylin and eosin (SigmaAldrich). All slides were evaluated by investigators blinded to the experimental conditions. The degrees of cell infiltration, synovial hyperplasia and bone erosion were scored on a scale of 0 (normal) to 4 (severe): 1 , mild changes; 2 , moderate changes; 3 , serve changes; and 4, total destruction of the joint architecture.

\section{Quantification of cytokines and autoantibodies}

The mice were sacrificed and the serum was harvested on experimental day 48 . The levels of cytokines (IL-6, IL-1 $\beta$, TNF- $a$, IL17, and IFN- $\gamma$; ExCell, Taichang, Jiangsu, China) and autoantibodies (anti-CII IgG; Chondrex, Redmond, WA, USA) were measured by using an enzyme-linked immunosorbent assay (ELISA) kit according to the manufacturer's instructions.

Sample preparation and the pharmacokinetics of kaempferol Healthy DBA/1J mice were fasted overnight; then, kaempferol was administered to the mice orally $(200 \mathrm{mg} / \mathrm{kg})$ or injected intraperitoneally $(20 \mathrm{mg} / \mathrm{kg})$. Blood samples $(60 \mu \mathrm{L})$ were collected in heparinized tubes at $0.25,0.5,1,2,4$, and $6 \mathrm{~h}$, and the plasma was obtained by centrifugation at $8000 \mathrm{r} / \mathrm{min}$ for $5 \mathrm{~min}$ and stored at $-70^{\circ} \mathrm{C}$ until analysis. In an independent experiment, tissue samples were harvested at $0.25,0.5$, and $2 \mathrm{~h}$, and $50 \mathrm{mg}$ samples of each tissue were accurately weighed, cut into slices, added to $0.5 \mathrm{~mL}$ of deionized water $(0.1 \mathrm{~g} / \mathrm{mL})$, and homogenized in an ice bath. Fecal samples were obtained at intervals of $0-24 \mathrm{~h}$ and homogenized with deionized water $(1: 10, \mathrm{w} / \mathrm{w})$.

Kaempferol and kaempferol-3-O-glucuronide were extracted by direct precipitation [17]. Briefly, $30 \mu \mathrm{L}$ of biological sample (plasma, tissue or fecal homogenate) was added to $300 \mu \mathrm{L}$ of acetonitrile containing IS (warfarin, $0.2 \mathrm{ng} / \mathrm{mL}$ ). The mixture was shaken and centrifuged twice at $30065 \times \mathrm{g}$ for $5 \mathrm{~min}$, and the supernatant was injected for analysis.

The levels of kaempferol and kaempferol-3-O-glucuronide in the biological samples were determined by a Shimadzu LC system with an Applied Biosystems/MDS Sciex API 5500-Qtrap triple quadrupole mass spectrometer. Chromatographic separation was achieved on a Waters XSelect HSS T3 column $(3.0 \mathrm{~mm} \times 50 \mathrm{~mm}$, $2.5 \mu \mathrm{m})$ at $40^{\circ} \mathrm{C}$. The mobile phase was composed of solvent
A (containing $0.1 \%$ formate, $\mathrm{v} / \mathrm{v}$ ) and solvent $\mathrm{B}$ (acetonitrile), with a gradient elution $(10.0 \%-20.0 \%$ B at $0.0-2.8 \mathrm{~min}, 20.0 \%-95.0 \%$ $B$ at $2.8-5.0 \mathrm{~min}$ maintained for $1.5 \mathrm{~min}$ and $95.0 \%-10.0 \% \mathrm{~B}$ at $6.5-7.5 \mathrm{~min}$ maintained at $10 \% \mathrm{~B}$ for $2.0 \mathrm{~min}$ at a flow rate of $0.3 \mathrm{~mL} / \mathrm{min}$ ). The $3 \mu \mathrm{L}$ samples were injected for analyses. The mass spectrometer was operated in negative ion electrospray mode. Quantification was obtained using the multiple reaction monitoring (MRM) acquisition mode by monitoring the precursor ion to product ion transitions of $\mathrm{m} / \mathrm{z} 285.2 \rightarrow 117.0$ for kaempferol, $462.0 \rightarrow 286.0$ for kaempferol-3-O-glucuronide and $307.2 \rightarrow 250.0$ for IS. The data acquisition and processing was performed using Analyst 1.5.2 Software (AB SCIEX, CA, USA). The linear ranges of kaempferol and kaempferol-3-O-glucuronide were $0.01-30 \mathrm{ng} / \mathrm{mL}$ and $1-1000 \mathrm{ng} / \mathrm{mL}$, respectively.

$16 \mathrm{~S}$ rRNA-assisted high-throughput sequencing analysis

Fecal samples from different groups were collected on day 48 and were immediately stored in liquid nitrogen. The total DNA was extracted using an E.Z.N.A. Stool DNA Kit. Then, the V3-V4 domain of the 16S rRNA gene was amplified using primers (341F: 5'-CCTAYGGGRBGCASCAG-3'; 806R: 5'-GGACTACNNGGGTAT (TAAT-3') and a Phanta Max Super-Fidelity DNA Polymerase Kit. Then, the PCR amplification product was recovered and quantified using a QuantiFluor ${ }^{\mathrm{TM}}$ fluorometer. The purified amplification products were mixed in equal amounts and connected through sequencing joints. Finally, a sequencing library was constructed, and sequencing was conducted on a HiSeq 2500 instrument with PE250 sequencing.

Metabolomic analysis of the gut contents

On day 48 after the first immunization, the cecal contents of the mice were recovered and freeze-dried. The samples were stored at $-80^{\circ} \mathrm{C}$ for further analysis. Approximately $10 \mathrm{mg}$ of freeze-dried cecal contents was homogenized with $200 \mu \mathrm{L}$ of water $(1: 2, \mathrm{w} / \mathrm{v})$. The mixture was centrifuged at $10000 \mathrm{r} / \mathrm{min}$ for $10 \mathrm{~min}$, and $50 \mu \mathrm{L}$ of supernatant was transferred to a new Eppendorf tube; then, $200 \mu \mathrm{L}$ of methanol (containing the internal standard $\left[{ }^{13} \mathrm{C}_{2}\right]-$ myristic acid $5 \mu \mathrm{g} / \mathrm{mL}$ ) was added and vortexed for 5 min and centrifuged at $20000 \times g$ for $10 \mathrm{~min}$ at $4^{\circ} \mathrm{C}$. A $100 \mu \mathrm{L}$ aliquot of the supernatant was dried. The following process was performed with the previously described GC-MS conditions for metabolomic analysis [18]. In brief, the temperature was initially set at $100^{\circ} \mathrm{C}$ for $5 \mathrm{~min}$ and then increased to $300^{\circ} \mathrm{C}$ over $25 \mathrm{~min}$. Once the temperature reached $300^{\circ} \mathrm{C}$, it was maintained for another $5 \mathrm{~min}$. The masses were scanned at $\mathrm{m} / \mathrm{z} 50-780$. The normalized peak area (normalized by IS) was introduced in SIMCA-P software for multivariate statistical analysis.

Statistical analysis

All data are expressed as the mean $\pm S D$. Differences among groups were evaluated by one-way ANOVA. $P$-values $<0.05$ were considered to indicate statistical significance.

\section{RESULTS}

Oral administration of kaempferol alleviated arthritis in mice with $\mathrm{CIA}$

To test the therapeutic effect of kaempferol after intragastric or intraperitoneal administration, an experimental arthritis model was induced by the administration of collagen with complete Freund's adjuvant. Mice gradually lost weight after the onset of arthritis, and the spleen indices were significantly increased compared with those of the normal mice. Oral treatment with kaempferol significantly prevented the decline in body weight, and kaempferol treatment caused the mice to regain their body weight starting on day 33. The spleen index was markedly decreased after intragastric administration (Fig. 1a, b). Paw swelling was observed on day 27 after the first immunization 
a

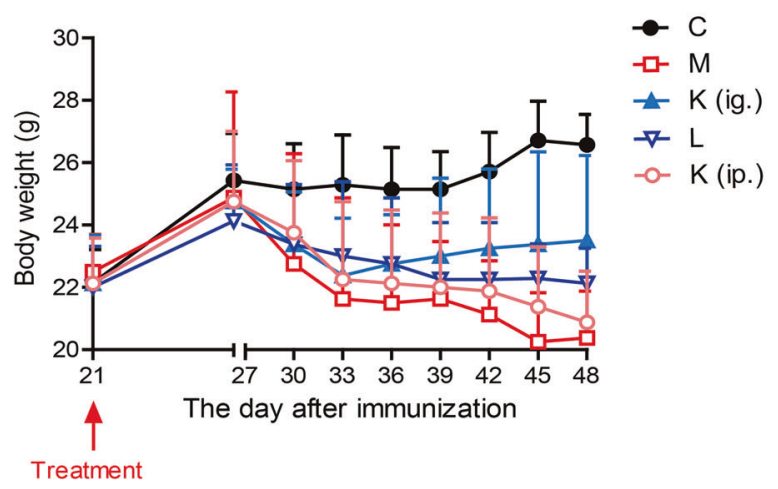

C

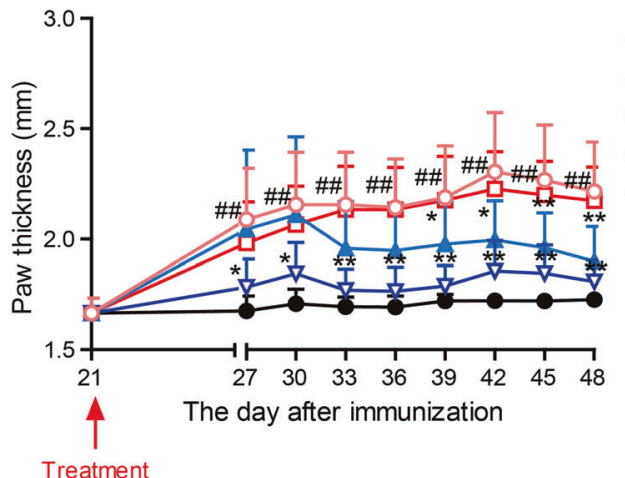

e

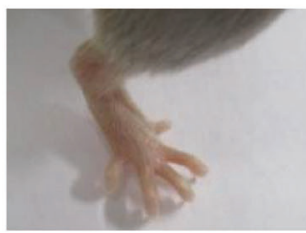

f

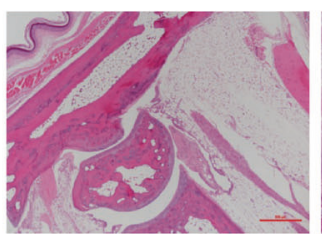

C
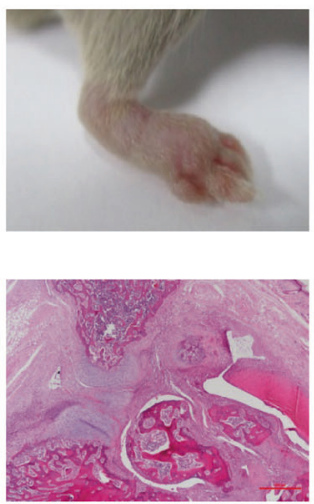

M

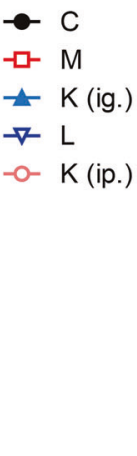

b

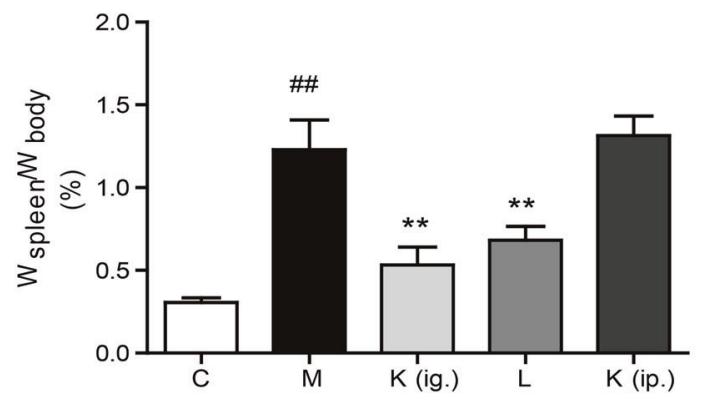

d
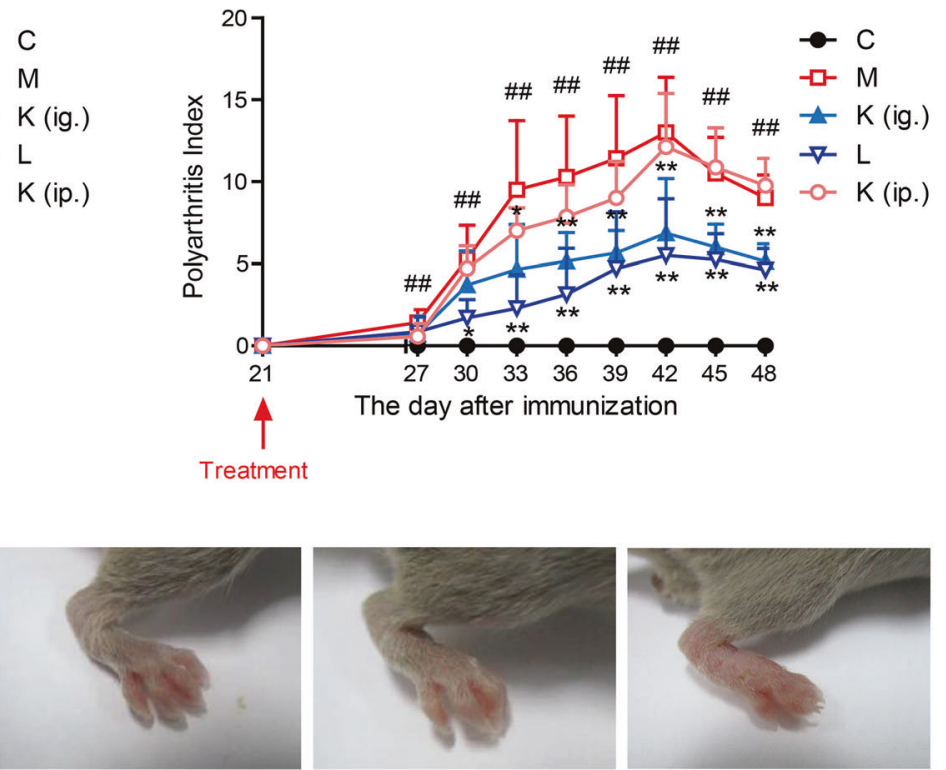

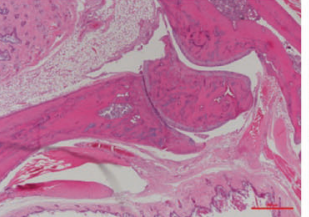

K (ig.)

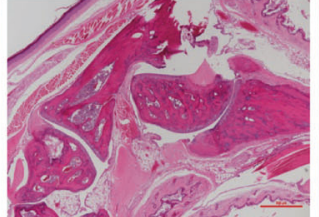

L

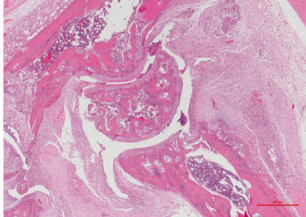

K (ip.)

Fig. 1 Oral treatment with kaempferol prevents arthritis in CIA mice. a Changes in the body weights of the mice; $\mathbf{b}$ The spleen index was calculated as the ratio of the spleen weight to the mouse body weight; $\mathbf{d}$ Polyarthritis index; $\mathbf{c}$, e Paw swelling; $\mathbf{f}$ Histological examination of ankle joint sections (H\&E staining). Mean \pm SD, $n=6$. C: control, M: model, K (ig.): oral administration of kaempferol, $\mathrm{K}$ (ip.): intraperitoneal injection of kaempferol, L: leflunomide. ${ }^{\# \#} P<0.01$ vs. $C ;{ }^{*} P<0.05,{ }^{* *} P<0.01$ vs. $M$

and reached a peak on day 42. Oral kaempferol treatment significantly ameliorated the CIA symptoms in mice and reduced the arthritis index and paw thickness (Fig. 1c-e). Histopathological examination by $\mathrm{H} \& \mathrm{E}$ staining showed that the ankle joint in $\mathrm{CIA}$ mice exhibited significant pathological changes, including inflammatory cell infiltration, cell proliferation, and disorderly cell arrangement. After the intragastric administration of kaempferol, the pathological changes were significantly reduced in comparison with those of the untreated mice (Fig. 1f). The positive control leflunomide also suppressed arthritis symptoms (Fig. 1a-f). However, there was little improvement in body weight, spleen index and paw swelling after the intraperitoneal injection of kaempferol (Fig. 1a-f). In summary, oral administration, instead of the intraperitoneal injection of kaempferol, exerted a distinct effect on arthritis.

Kaempferol depressed inflammatory cytokine and pathogenic antibody secretion in CIA mice

In comparison with those of the normal mice, the circulating levels of IL-6, TNF- $a$, IL-1 $\beta$, and IFN- $\gamma$ were much higher in arthritic mice (Fig. 2a-d). The levels of proinflammatory cytokines were significantly reduced in mice orally treated with kaempferol and leflunomide (Fig. 2a-d). However, no significant difference was observed after the parenteral administration of kaempferol in the treated group relative to the model group (Fig. 2a-d). Additionally, the total anti-CII lgG level in the plasma was markedly decreased 


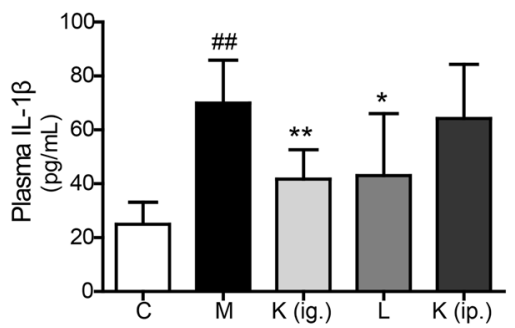

b

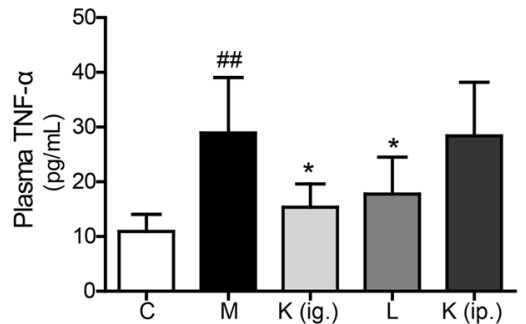

C

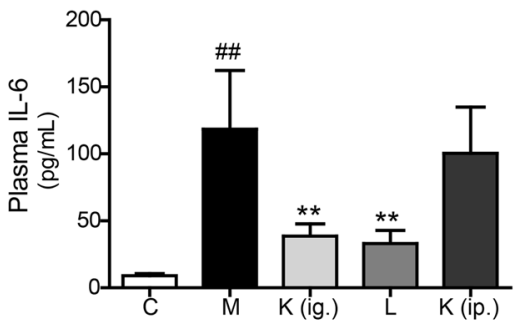

d

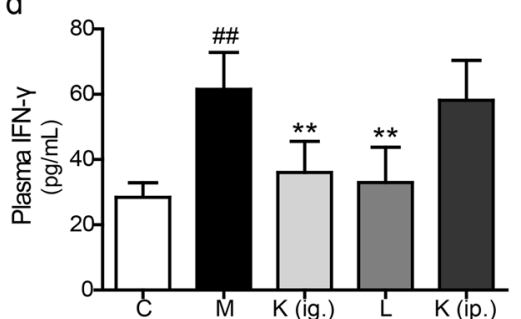

e

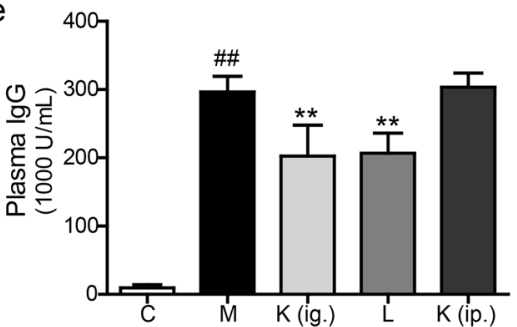

Fig. 2 Kaempferol decreases inflammatory cytokine and pathogenic antibody secretion in CIA mice. The levels of proinflammatory cytokines a IL-1 $\beta$, b TNF- $\alpha$, c IL-6, and d IFN- $\gamma$ and e anti-CIl antibodies. Mean \pm SD, $n=6$. C: control, M: model, $\mathrm{K}$ (ig.): oral administration of kaempferol, $\mathrm{K}$ (ip.): intraperitoneal injection of kaempferol, L: leflunomide. ${ }^{\# \#} P<0.01$ vs. $C ;{ }^{*} P<0.05,{ }^{* *} P<0.01$ vs. $M$<smiles>O=c1c(O)c(-c2ccc(O)cc2)oc2cc(O)cc(O)c12</smiles>

kaempferol $\mathrm{R}=\mathrm{H}$ kaempferol-3-O-glucuronide $\mathrm{R}=$ Glucuronic acid b

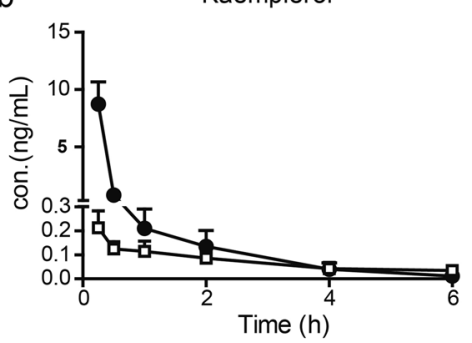

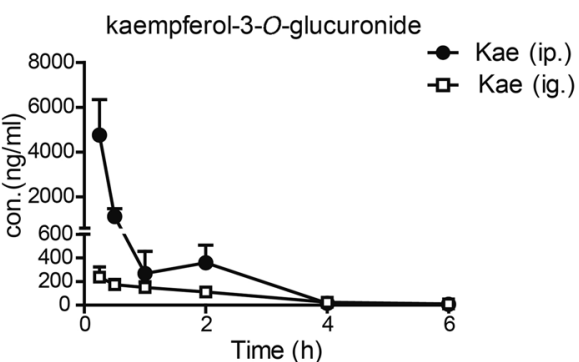

d

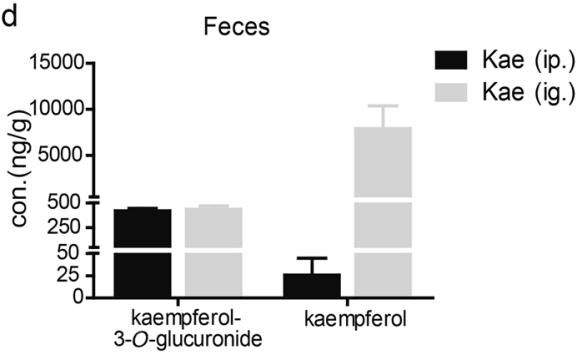

Fig. 3 Pharmacokinetic profile of kaempferol and kaempferol-3-O-glucuronide after the oral administration (200 mg/kg) or intraperitoneal injection $(20 \mathrm{mg} / \mathrm{kg})$ of kaempferol. a The chemical structure of kaempferol and kaempferol-3-O-glucuronide. b Mean plasma concentration-time profile of kaempferol and kaempferol-3-O-glucuronide, $n=8$. c Splenic distribution profiles of kaempferol and kaempferol-3-O-glucuronide, $n=6$. d The concentration of kaempferol and kaempferol-3-O-glucuronide in feces collected in $24 \mathrm{~h}$. Mean \pm SD, $n=6$. K (ig.): oral administration of kaempferol, K (ip.): intraperitoneal injection of kaempferol

both in mice who had been intragastrically (ig.) administered kaempferol and in leflunomide-treated mice compared to that of mice who had received an intraperitoneal (ip.) injection of kaempferol (Fig. 2e).

Pharmacokinetics of kaempferol and its primary metabolite kaempferol-3-O-glucuronide

After oral administration, kaempferol undergoes phase II glucuronidation in vivo; one of the primary metabolites identified in this process is kaempferol-3-O-glucuronide, which is commercially available [17]. (Fig. 3a). To assess the levels of kaempferol and kaempferol-3-O-glucuronide in vivo, the pharmacokinetic properties were investigated via intragastric and intraperitoneal routes. In the plasma, high levels of the parent drug kaempferol and its metabolite were observed after the intraperitoneal injection of kaempferol. However, relatively low levels were observed in plasma after intragastric administration (Fig. 3b). In the spleen, the levels of kaempferol and kaempferol-3-Oglucuronide were relatively low after intragastric and intraperitoneal administration (Fig. 3c). Conversely, the level of kaempferol in the gut was greatly concentrated (Fig. 3d) after the oral administration of kaempferol. We hypothesized that the high level of kaempferol in the gut may be involved in the therapeutic effects of kaempferol. 


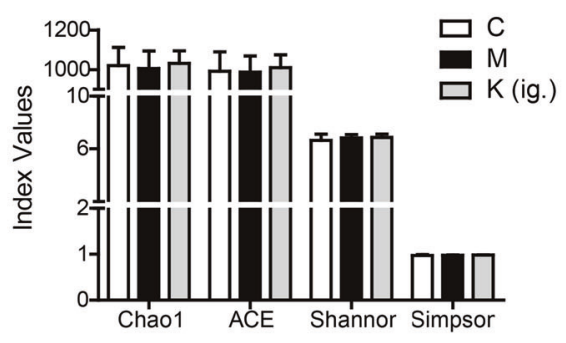

b

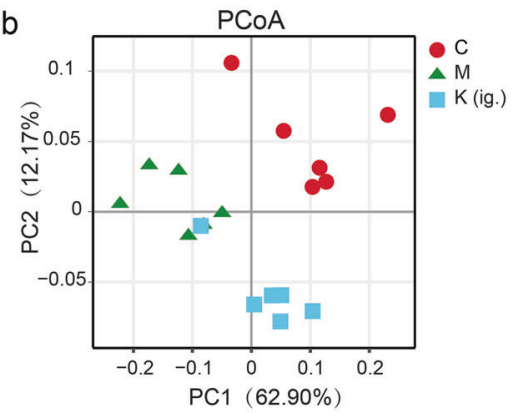

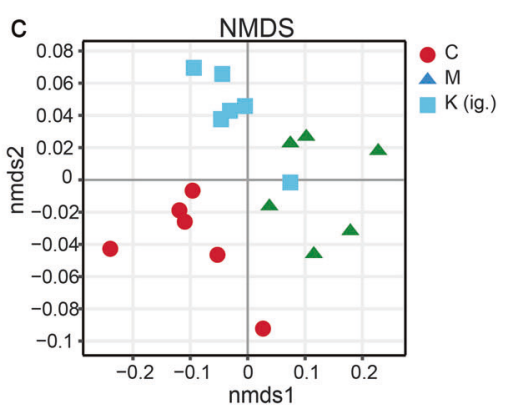

d

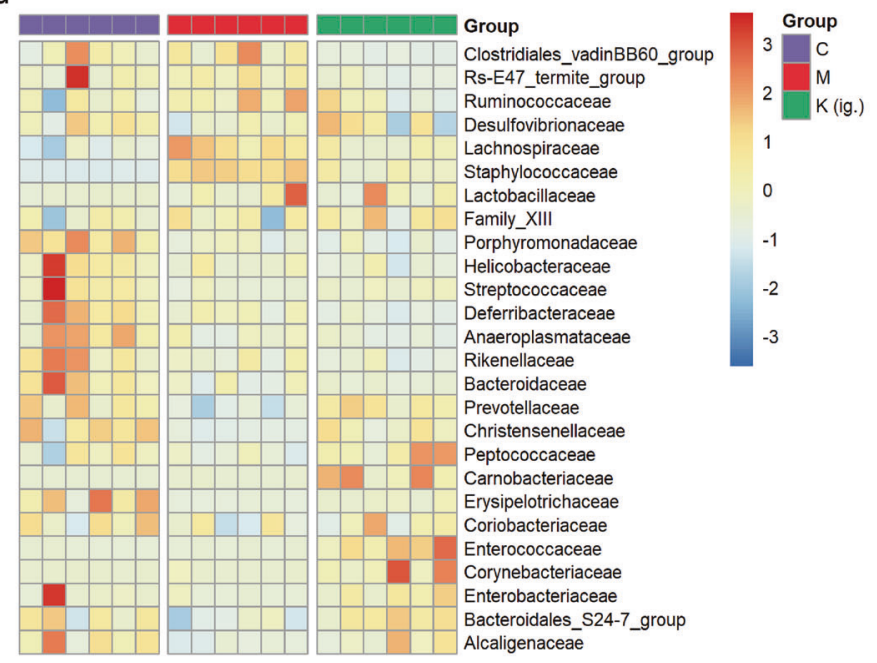

e
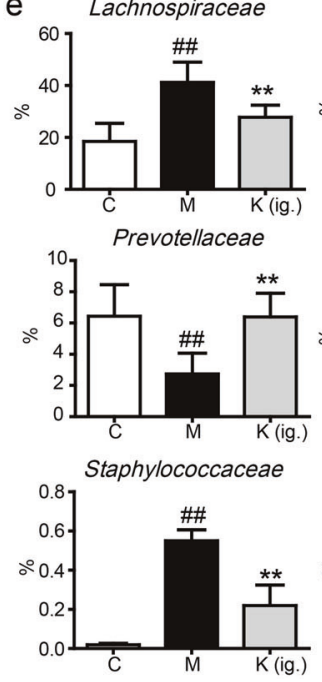

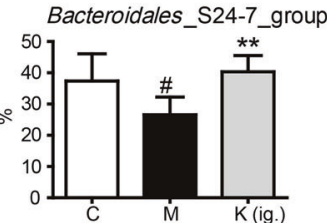

Erysipelotrichaceae

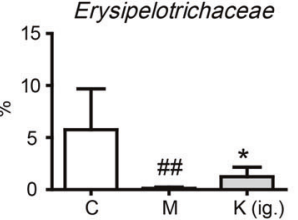

Alcaligenaceae

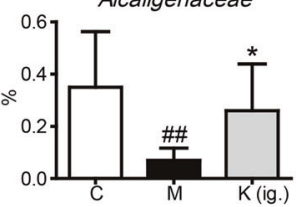

Fig. 4 Kaempferol treatment modulates intestinal microbial composition in CIA mice. a $\alpha$-Diversity assessed by Chao, Shannon, Simpson, and ACE indices in the control, model and kaempferol groups. b, c A plot of unconstrained principal coordinate analysis and nonmetric multidimensional scaling based on weighted UniFrac distances. d The microbial communities at the family level. e The representative microbial community was altered at the family level in the kaempferol treatment group compared with that of the CIA treatment group. Mean $\pm S D, n=6$. C: control, M: model, K (ig.): oral administration of kaempferol. ${ }^{\#} P<0.05,{ }^{\# \#} P<0.01$ vs. C; ${ }^{*} P<0.05,{ }^{* *} P<0.01$ vs. M

Kaempferol rebalanced the dysbiosis of the gut microbiota in $\mathrm{CIA}$ mice

Considering the much higher level of kaempferol in the gut after oral administration, we speculated that kaempferol exerts its antiarthritis effect by affecting the microbiota. The a-diversity of the gut microbiome in model mice did not show significant differences from that of the control mice; kaempferol treatment marginally affected the a-diversity of the gut microbiome (Fig. 4a). However, both principal coordinate analysis (PCoA) and nonmetric multidimensional scaling (NMDS) of OTUs scored by weighted UniFrac dissimilarity revealed that the microbiota of the CIA mice deviated from that of the normal control mice, with a clear separation (Fig. 4b, c). The administration of kaempferol reshaped the microbiota composition, with some of the microbiota becoming rebalanced after treatment with kaempferol (Fig. 4b-d).

At the family level, 14 bacteria significantly changed in the arthritis samples compared with the control samples $(P<0.05)$; furthermore, treatment with kaempferol regulated the levels of Lachnospiraceae, Bacteroidales_S24-7_group, Prevotellaceae, Erysipelotrichaceae, Staphylococcaceae, and Alcaligenaceae (Fig. 4e). These data indicate that oral treatment with kaempferol rebalances the intestinal gut flora in CIA mice.

Kaempferol-mediated modulation of metabolites in CIA mouse fecal samples

The deconvolution of the metabolic features in the total ion chromatograms of fecal samples revealed 128 peaks, of which 101 metabolites were authentically identified (Fig. 5). The PLS-DA model (Fig. 6a) showed that samples from each of the three groups of samples tended to cluster closely, indicating that the metabolic features within each group were similar. Furthermore, the three groups of samples were scattered, indicating that the metabolic features of the three groups of samples were different. The OPLS analysis (Fig. 6b, c) showed a good separation and difference between the CIA mice and the normal control mice, and the CIA mice and the kaempferol-treated mice, respectively. The SUS plot highlighted the metabolites that greatly contributed to the model (Fig. 6d). In total, 44 metabolites, including organic acids, amino acids, saccharides, and lipids, were significantly different in the feces of the CIA mice compared to the normal control mice. Treatment with kaempferol reversed $54.5 \%$ ( 24 molecules out of the 44 metabolites, Supplementary Table S1) of the metabolites toward the normal levels (Fig. 6e). Metabolic pathway analysis showed that these metabolites are involved in the metabolism of tryptophan, fatty acids, and secondary bile acids, and in energy production (Fig. 7). The oral administration of kaempferol regulates microbial metabolism in CIA mice.

\section{DISCUSSION}

Previous studies have reported that kaempferol relieves the symptoms of arthritis in mice [13] and suppresses the formation of osteoclasts, and the migration and invasion of fibroblast-like synoviocytes in vitro $[13,15,16]$. In this study, we confirmed that oral treatment with kaempferol significantly ameliorated arthritis 


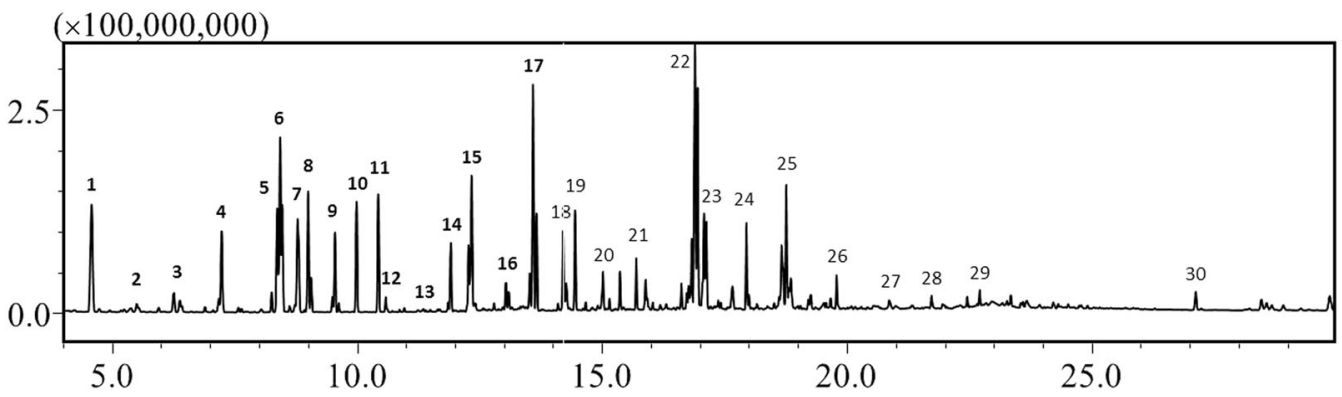

Fig. 5 Typical GC/MS chromatogram of the extracted molecules in feces. Typical compounds are identified and numbered as follows: 1. Lactate; 2. Oxalic acid; 3. 2-Aminobutyric acid; 4. L-Norvaline; 5. L-Alanine; 6. Phosphoric acid; 7. L-Isoleucine; 8. Glycine; 9. Uracil; 10. Serine; 11. L-Threonine; 12. Thymine; 13. Homoserine; 14. Malic acid; 15. L-Aspartic acid; 16. 2-ketoglutaric acid; 17. L-Glutamic acid; 18. Ribose; 19. Arabinose; 20. Fucose; 21. Hypoxanthine; 22. Glucose; 23. Mannose; 24. Xanthine; 25. Myo-Inositol; 26. L-Tryptophan; 27. Arachidonic acid; 28. Uridine; 29. Inosine; and 30. Cholesterol

symptoms and decreased inflammatory cytokine levels in CIA mice. Unexpectedly, the intraperitoneal injection of kaempferol showed marginal efficacy in the CIA model mice. To our surprise, intraperitoneal injection of kaempferol achieved a much higher in vivo level of kaempferol and its primary metabolite, kaempferol3-O-glucuronide, than intragastric administration. In other words, despite the high levels of kaempferol-3-O-glucuronide and kaempferol in the circulation after intraperitoneal injection, the parenteral administration of kaempferol showed little improvement in arthritis. The distinct PK-PD discrepancy greatly challenges the underlying mechanism and the kaempferol-targeted organs and tissues.

Although the levels of kaempferol-3-O-glucuronide and kaempferol were relatively low in the circulating blood and spleen, we found high levels of kaempferol in the gut after oral administration. Because the gut microbiota play a crucially important role in anti-inflammatory immunity, [19-22] the gut flora were examined in the CIA model mice. The 16S rRNA sequence analysis revealed the perturbed composition of intestinal microbiota in the CIA mice, while kaempferol treatment rebalanced the intestinal microbial community. In CIA mouse fecal samples, Lachnospiraceae and Staphylococcaceae were enriched, and the abundance of Bacteroidales_S24-7_group, Prevotellaceae, Bacteroidaceae, Erysipelotrichaceae, Alcaligenaceae, and Christensenellaceae was diminished at the family level. Consistently, previous studies have suggested that Lachnospiraceae, Staphylococcus aureus (S. aureus), Prevotellaceae, and Bacteroidales_S24-7_group are associated with arthritis [23-25]. It has been reported that Bacteroidales_S247_group is significantly decreased in CIA mice, [22] and this bacteria are associated with the production of anti-inflammation molecules, such as acetate and propionate [26]. To some extent, kaempferol reshaped the gut flora and primarily modulated the abundance of Lachnospiraceae, Bacteroidales_S24-7_group, Prevotellaceae, Erysipelotrichaceae, Staphylococcaceae, and Alcaligenaceae, suggesting that the anti-arthritis effect of kaempferol involves the intestinal microbiota.

The gut microbiota not only influence the maturation and development of the host immune system but also influence host metabolic homeostasis by cross-communication via microbial metabolites or cometabolites. In this study, a significantly perturbed metabolism was observed in the fecal extracts of CIA mice. For example, tryptophan metabolism plays an important role in microbiota-host crosstalk in health and disease [27]. In the present study, reduced tryptophan and elevated indole-3-acetic acid in the CIA group demonstrated that tryptophan metabolism was enhanced in mice with arthritis. Indole-3-acetic acid, the metabolite of tryptophan in the gut, is a ligand for AhR (aryl hydrocarbon receptor), which regulates the immune response and intestinal homeostasis $[27,28]$. Kaempferol treatment efficiently reversed the tryptophan metabolism, decreased the indole-3- acetic acid level and increased the tryptophan level, which further suggests that kaempferol modulates the gut flora.

Glucose, fructose, and the key intermediate of the citric acid cycle a-ketoglutaric acid are important factors that regulate $T$ cell activation [29] and differentiation [30]. Treatment with kaempferol efficiently reduced the levels of fructose, glucose, and aketoglutaric acid in the gut, suggesting that kaempferol may modulate energy metabolism and affect $T$ cell properties. In contrast to the upregulated serum fatty acid levels in CIA rats in a previous study [31], the levels of many fatty acids, such as palmitoleic acid, palmitic acid, linoleic acid, and oleic acid, were significantly decreased in the feces of CIA mice. However, kaempferol reversed the decreased levels of these fatty acids, indicating the modulation of the gut flora and the turnover of intestinal lipids.

Bile acids are closely associated with immune inflammation $[32,33]$. In addition, their metabolism and turnover exclusively involves the gut microbiota. In addition, primary bile acids are transformed into secondary bile acids by the means of the gut flora. Arthritis significantly increases the levels of cholic acid (DC) and deoxycholic acid (DOCA) and decreases the level of glycocholic acid (GCA). DOCA is a secondary bile acid predominantly metabolized by the intestinal microbiota, and a previous study reported that DOCA induces severe inflammation [34]. Treatment with kaempferol decreased the DOCA level and had little effect on DC and GCA levels, again indicating that kaempferol exerts anti-arthritis effects by modulating the gut microbiota.

\section{CONCLUSION}

Intraperitoneal injection achieves high plasma levels of kaempferol and its primary metabolite, yet marginal effects are observed. Orally administered kaempferol has relatively low bioavailability and in vivo exposure, yet it shows distinct anti-arthritis activity. The high level of kaempferol in the gut after oral administration reshapes the intestinal microbial community and modulates the microbiota-mediated metabolism of tryptophan, fatty acids and secondary bile acids and energy production, which may contribute to the effectiveness of kaempferol in RA.

\section{ACKNOWLEDGEMENTS}

This study was financially supported by the National Natural Science Foundation of China (81530098 and 81573495), the National Key Special Project of Science and Technology for Innovation Drugs of China (2015ZX09501001 and 2017ZX09301013), the Project of the University Collaborative Innovation Center of Jiangsu Province (Modern Chinese Medicine Center and Biological Medicine Center) and the Natural Science Foundation of Shanghai (16ZR1434300). 


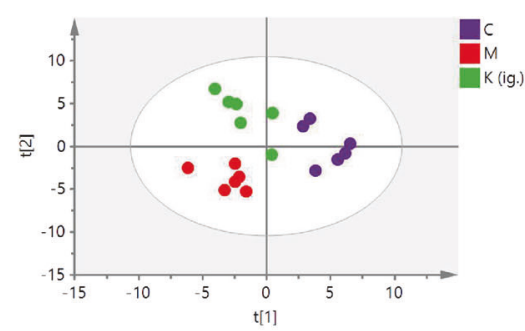

C

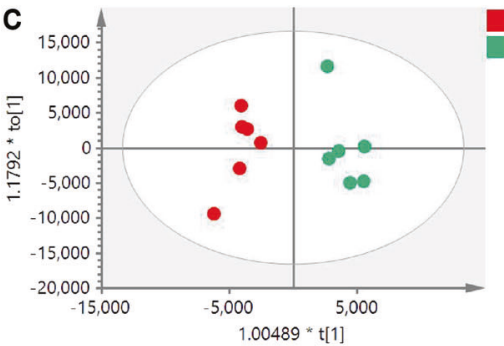

b
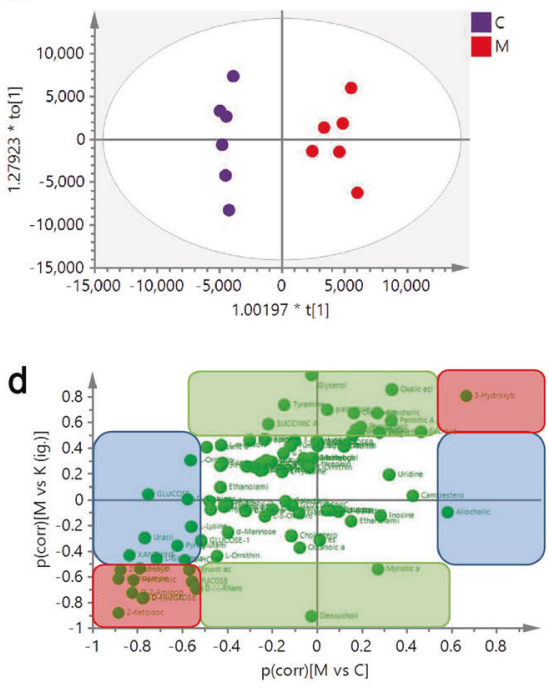

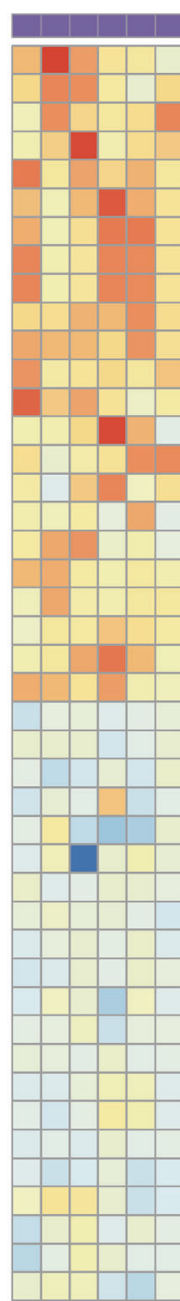

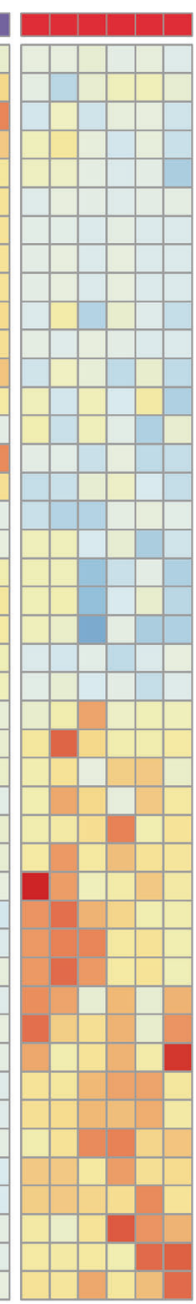

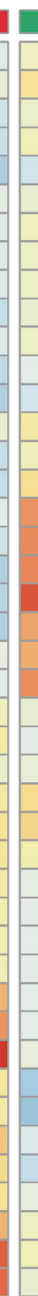

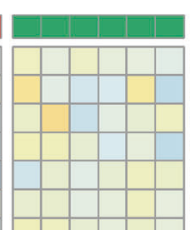

\section{Group}

Propanedioic acid Succinate

L-Alanine-2TMS

3-Hydroxyisovaleric acid

Ethanolamine

Palmitoleic acid

Linoleic acid

11-cis-Octadecenoic acid

Oleic Acid

3-Hydroxyvaleric acid

Glycerol

Oxalic acid-2TMS

Hydracrylic acid

Monomethylphosphate

Palmitic Acid

L-Tryptophan

Prost-13-en-1-oic acid

L-Threonine

Fumarate

Malic acid

Myo-Inositol

3-Hydroxybutyric acid

GCA

CA

2-Aminobutyric acid

Benzoic acid

L-Glutamic acid

Thymine

Mannose

Maltose

3-Methyl-2-ketobutyric acid

Fucose

Rhamnose

2-Ketoisocaproic acid

2-Ketoglutaric acd

Fructose

Glucuronic acid

Galactaric acid

1H-Indole-3-acetic acid

DOCA

2-Hydroxybutyric acid

Glucose

Niacin
Homoserin

Fig. 6 Kaempferol treatment modulates the metabolites in fecal samples of CIA mice. Metabolic patterns of the three groups of mice based on the multivariate statistical analysis of the GC/MS data. $\mathbf{a}$ The score plots of the three groups and their PLS-DA models for fecal data; $\mathbf{b}$ CIA vs. normal groups and their OPLS-DA models for fecal data; c kaempferol treatment vs. CIA treatment and the OPLS-DA for fecal data; d SUS plot of fecal samples correlating the OPLS-DA models of CIA versus the control ( $x$-axis) and CIA versus kaempferol treatment ( $y$-axis). Accordingly, the variables in the lower left corners and higher right corners are the compounds whose levels were reversed after kaempferol treatment of CIA mice (red box). However, metabolites located along the axes were specifically altered in the model group (blue boxes) and the normal group (green boxes). e Heatmap visualizing the intensities of differential metabolites in the fecal samples ( $n=6)$. C: control, M: model, K (ig.): oral administration of kaempferol 


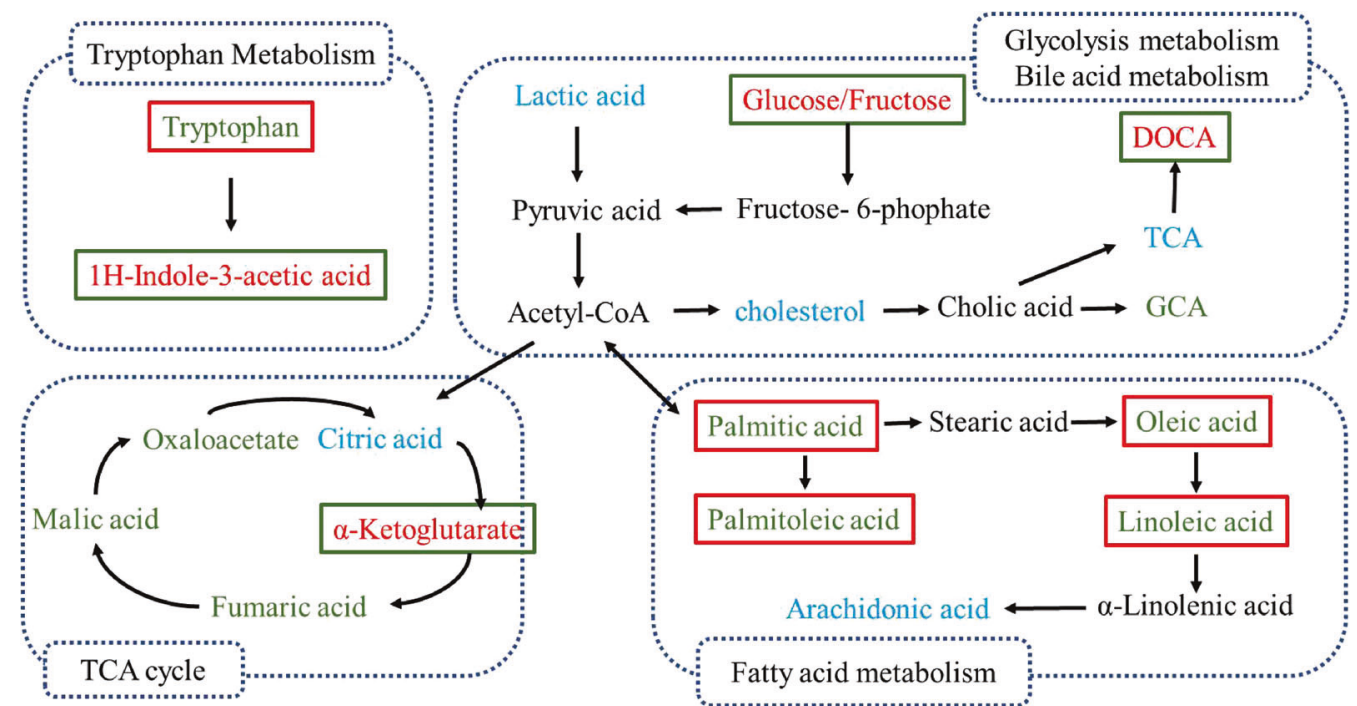

Red Up-regulated in $\mathrm{M}$ versus $\mathrm{C}$ group

Green Down-regulated in $\mathrm{M}$ versus $\mathrm{C}$ group

Blue No significant change in $\mathrm{M}$ versus $\mathrm{C}$ group

Black Not detected in current samples
Up-regulated in $\mathrm{K}(\mathrm{ig}$.) versus $\mathrm{M}$ group

Down-regulated in $\mathrm{K}$ (ig.) versus $\mathrm{C}$ group

Fig. 7 Typical metabolites and associated pathways modulated by kaempferol treatment

\section{AUTHOR CONTRIBUTIONS}

JYA, GJW, and CXL designed this study; $L X A, F F, Q Q$, and RBS performed the experiments, analyzed the data, and prepared the paper; JYA, GJW, and CXL revised the paper; and SHG and ZZD edited the English. All authors read and approved the final paper.

\section{ADDITIONAL INFORMATION}

The online version of this article (https://doi.org/10.1038/s41401-019-0279-8) contains supplementary material, which is available to authorized users.

\section{REFERENCES}

1. Scott DL, Wolfe F, Huizinga TW. Rheumatoid arthritis. Lancet. 2010;376: 1094-1108.

2. Smolen JS, Aletaha D, McInnes IB. Rheumatoid arthritis. Lancet. 2016;388:2023-38.

3. Jin J, Chang $Y$, Wei W. Clinical application and evaluation of anti-TNF-alpha agents for the treatment of rheumatoid arthritis. Acta Pharmacol Sin. 2010;31: 1133-40.

4. Shi W, Wang YM, Li SL, Yan M, Li D, Chen BY, et al. Risk factors of adverse drug reaction from non-steroidal anti-inflammatory drugs in Shanghai patients with arthropathy. Acta Pharmacol Sin. 2004:25:357-65.

5. He D. Y., Dai S. M. Anti-inflammatory and immunomodulatory effects of Paeonia lactiflora Pall., a traditional Chinese herbal medicine. Front Pharmacol. 2011;2:10.

6. Wang J, Li Y, Yang Y, Du J, Zhao M, Lin F, et al. Systems pharmacology dissection of multiscale mechanisms of action for herbal medicines in treating Rheumatoid Arthritis. Mol Pharmacol. 2017;14:3201-17.

7. Yang $Y$, Zhang $X, X u M$, Wu X, Zhao F, Zhao C. Quercetin attenuates collageninduced arthritis by restoration of Th17/Treg balance and activation of heme oxygenase 1-mediated anti-inflammatory effect. Int Immunopharmacol. 2018;54:153-62.

8. Devi KP, Malar DS, Nabavi SF, Sureda A, Xiao J, Nabavi SM, et al. Kaempferol and inflammation: from chemistry to medicine. Pharmacol Res. 2015;99:1-10.

9. Rajendran P, Rengarajan T, Nandakumar N, Palaniswami R, Nishigaki Y, Nishigaki I. Kaempferol, a potential cytostatic and cure for inflammatory disorders. Eur J Med Chem. 2014;86:103-12.

10. Wang J, Fang X, Ge L, Cao F, Zhao L, Wang Z, et al. Antitumor, antioxidant and anti-inflammatory activities of kaempferol and its corresponding glycosides and the enzymatic preparation of kaempferol. PLoS ONE. 2018;13:e0197563.
11. Kundu S, Ghosh P, Datta S, Ghosh A, Chattopadhyay S, Chatterjee M. Oxidative stress as a potential biomarker for determining disease activity in patients with rheumatoid arthritis. Free Radic Res. 2012;46:1482-9.

12. Choy EHS, Panayi GS. Mechanisms of disease: cytokine pathways and joint inflammation in rheumatoid arthritis. New Engl J Med. 2001;344:907-16.

13. Pan D, Li N, Liu $Y, X u Q$, Liu Q, You $Y$, et al. Kaempferol inhibits the migration and invasion of rheumatoid arthritis fibroblast-like synoviocytes by blocking activation of the MAPK pathway. Int Immunopharmacol. 2018;55: 174-82.

14. Lattig J, Bohl M, Fischer P, Tischer S, Tietbohl C, Menschikowski M, et al. Mechanism of inhibition of human secretory phospholipase $\mathrm{A} 2$ by flavonoids: rationale for lead design. J Comput Aid Mol Des. 2007;21:473-83.

15. Lee WS, Lee EG, Sung MS, Yoo WH. Kaempferol inhibits IL-1beta-stimulated, RANKL-mediated osteoclastogenesis via downregulation of MAPKs, c-Fos, and NFATc1. Inflammation. 2014;37:1221-30.

16. Yoon HY, Lee EG, Lee H, Cho IJ, Choi YJ, Sung MS, et al. Kaempferol inhibits IL1beta-induced proliferation of rheumatoid arthritis synovial fibroblasts and the production of COX-2, PGE2 and MMPs. Int J Mol Med. 2013;32:971-7.

17. Zabela V, Sampath C, Oufir M, Moradi-Afrapoli F, Butterweck V, Hamburger M. Pharmacokinetics of dietary kaempferol and its metabolite 4-hydroxyphenylacetic acid in rats. Fitoterapia. 2016;115:189-97.

18. He J, Gao HX, Yang N, Zhu XD, Sun RB, Xie Y, et al. The aldose reductase inhibitor epalrestat exerts nephritic protection on diabetic nephropathy in $\mathrm{db} / \mathrm{db}$ mice through metabolic modulation. Acta Pharmacol Sin. 2019;40:86-97.

19. Horta-Baas G, Romero-Figueroa M. D, Montiel-Jarquin A. J, Pizano-Zarate M. L, Garcia-Mena J, Ramirez-Duran N. Intestinal dysbiosis and rheumatoid arthritis: a link between gut microbiota and the pathogenesis of rheumatoid arthritis. J Immunol Res. 2017;2017:1-13.

20. Zhang $X$, Zhang DY, Jia $H J$, Feng $Q$, Wang $D H$, Liang $D$, et al. The oral and gut microbiomes are perturbed in rheumatoid arthritis and partly normalized after treatment. Nat Med. 2015;21:895-905.

21. Rogier R, Evans-Marin H, Manasson J, van der Kraan P. M, Walgreen B, Helsen MM, et al. Alteration of the intestinal microbiome characterizes preclinical inflammatory arthritis in mice and its modulation attenuates established arthritis. Sci Rep. 2017;7:15613.

22. Jubair WK, Hendrickson JD, Severs EL, Schulz HM, Adhikari S, Ir D, et al. Modulation of inflammatory arthritis in mice by gut microbiota through mucosal inflammation and autoantibody generation. Arthritis Rheumatol. 2018;70: 1220-33.

23. Rehaume LM, Matigian N, Mehdi AM, Lachner N, Bowerman KL, Daly J, et al. IL-23 favours outgrowth of spondyloarthritis-associated pathobionts and suppresses host support for homeostatic microbiota. Ann Rheum Dis. 2019;78:494-503. 
24. Baranwal G, Mohammad M, Jarneborn A, Reddy BR, Golla A, Chakravarty S, et al. Impact of cell wall peptidoglycan O-acetylation on the pathogenesis of Staphylococcus aureus in septic arthritis. Int J Med Microbiol. 2017;307:388-97.

25. Aggarwal A, Sarangi AN, Gaur P, Shukla A, Aggarwal R. Gut microbiome in children with enthesitis-related arthritis in a developing country and the effect of probiotic administration. Clin Exp Immunol. 2017;187:480-9.

26. Ormerod KL, Wood DL, Lachner N, Gellatly SL, Daly JN, Parsons JD, et al. Genomic characterization of the uncultured Bacteroidales family S24-7 inhabiting the guts of homeothermic animals. Microbiome. 2016;4:36.

27. Agus A, Planchais J, Sokol H. Gut microbiota regulation of tryptophan metabolism in health and disease. Cell Host Microbe. 2018;23:716-24.

28. Alexeev EE, Lanis JM, Kao DJ, Campbell EL, Kelly CJ, Battista KD, et al Microbiota-derived indole metabolites promote human and murine intestinal homeostasis through regulation of interleukin-10 receptor. Am J Pathol. 2018; 188:1183-94.

29. Jacobs SR, Herman CE, Maclver NJ, Wofford JA, Wieman HL, Hammen JJ, et al. Glucose uptake is limiting in $\mathrm{T}$ cell activation and requires CD28- mediated Akt-dependent and independent pathways. J Immunol. 2008;180: 4476-86.

30. Klysz D, Tai XG, Robert PA, Craveiro M, Cretenet G, Oburoglu L, et al. Glutaminedependent alpha-ketoglutarate production regulates the balance between $\mathrm{T}$ helper 1 cell and regulatory T cell generation. Sci Signal. 2015;8:ra97-ra97.

31. Shan JJ, Peng LX, Qian WJ, Xie T, Kang A, Gao B, et al. Integrated serum and fecal metabolomics study of collagen-induced arthritis rats and the therapeutic effects of the zushima tablet. Front Pharmacol. 2018;9:891

32. Guo CS, Xie SJ, Chi ZX, Zhang JH, Liu YY, Zhang L, et al. Bile acids control inflammation and metabolic disorder through inhibition of NLRP3 inflammasome. Immunity. 2016;45:802-16.

33. Duboc H, Rajca S, Rainteau D, Benarous D, Maubert MA, Quervain E, et al. Connecting dysbiosis, bile-acid dysmetabolism and gut inflammation in inflammatory bowel diseases. Gut. 2013;62:531-9.

34. Chung SJ, Lee $\mathrm{CH}$, Lee HS, Kim ST, Sohn UD, Park ES, et al. The role of phosphatidylcholine and deoxycholic acid in inflammation. Life Sci. 2014; 108:88-93. 